\title{
An Overview of the Guided Parafoil System Derived from X-38 Experience
}

\author{
Jenny M. Stein ${ }^{*}$, Chris M. Madsen ${ }^{\dagger}$, and Alan L. Strahan ${ }^{\ddagger}$ \\ NASA Johnson Space Center, Houston, State, 77058
}

\begin{abstract}
The NASA Johnson Space Center built a $4200 \mathrm{ft}^{2}$ parafoil for the U.S. Army Natick Soldier Center to demonstrate autonomous flight using a guided parafoil system to deliver $10,000 \mathrm{lbs}$ of useable payload. The parafoil's design was based upon that developed during the X-38 program. The drop test payload consisted of a standard 20 -foot Type $V$ airdrop platform, a standard 12-foot weight tub, a $60 \mathrm{ft}$ drogue parachute, a $4200 \mathrm{ft}^{2}$ parafoil, an instrumentation system, and a Guidance, Navigation, and Control (GN\&C) system. Instrumentation installed on the load was used to gather data to validate simulation models and preflight loads predictions and to perform post flight trajectory and performance reconstructions. The GN\&C system, developed during NASA's $X-38$ program, consisted of a flight computer, modems for uplink commands and downlink data, a compass, laser altimeter, and two winches. The winches were used to steer the parafoil and to perform the dynamic flare maneuver for a soft landing. The laser was used to initiate the flare. The GN\&C software was originally provided to NASA by the European Space Agency. NASA incorporated further software refinements based upon the $\mathrm{X}-\mathbf{3 8}$ flight test results. Three fullscale drop tests were conducted, with the third being performed during the Precision Airdrop Technology Conference and Demonstration (PATCAD) Conference at the U.S. Army Yuma Proving Ground (YPG) in November of 2003. For the PATCAD demonstration, the parafoil and GN\&C software and hardware performed well, concluding with a good flare and the smallest miss distance ever experienced in NASA's parafoil drop test program.

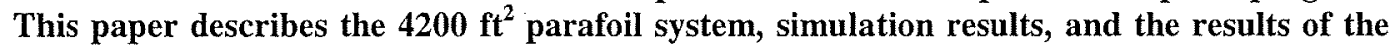
drop tests.
\end{abstract}

\section{Nomenclature}

$\begin{array}{ll}A D P & =\text { air data probe } \\ C R V & =\text { crew return vehicle } \\ C_{D} & =\text { coefficient of drag } \\ C G & =\text { center of gravity } \\ C_{L} & =\text { coefficient of lift } \\ D O F & =\text { degree of freedom } \\ D S S & =\text { decelerator system simulation } \\ E M C & =\text { energy management circle } \\ f^{2} & =\text { square foot } \\ G N C & =\text { guidance, navigation and control system } \\ G P S & =\text { global positioning system } \\ L D D & =\text { lift over drag }\end{array}$

\footnotetext{
* Aerospace Engineer, Exploration Systems Engineering Office/EX3, AIAA Associate Fellow.

${ }^{\dagger}$ Aerospace Engineer, Aeroscience and Flight Mechanics Division/EG, Member AIAA.

* Aerospace Engineer, Aeroscience and Flight Mechanics Division/EG, Member AIAA.
} 


$\begin{array}{ll}\text { LVAD } & =\text { low velocity air drop } \\ M S L & =\text { mean sea level } \\ P A T C A D & =\text { precision airdrop technology conference and demonstration conference } \\ P D S & =\text { parafoil dynamics simulator } \\ P G N C & =\text { parafoil guidance, navigation and control system } \\ \text { pSf } & =\text { pounds per square foot } \\ R A & =\text { rigging angle } \\ Y P G \quad & =\text { Yuma Proving Ground }\end{array}$

\section{Introduction}

The purpose of NASA's X-38 program was to develop a 25,000 lb crew return vehicle (CRV) with a sevenperson capacity to replace the Soyuz spacecraft currently used on the International Space Station. Figure 1 is a photograph of the spacecraft that was being built for an orbital flight test.

A lifting body shape was selected for the CRV because it has a large cross range capability for re-entry from Earth orbit. However, a lifting body has a high landing speed of 250 knots, requiring long runways and intensive pilot training. To reduce the CRV landing speeds to 40 knots, the program designed the landing system around a parafoil capable of performing a dynamic flare. The parafoil can be autonomously or manually steered to a pre-determined landing target, turned into the wind, flared and landed using a parafoil guidance, navigation and control system (PGNC). Due to the parafoil's relatively high lift to drag ratio, the parafoil also has good ground wind penetration for landing. Another advantage of using a parafoil landing system is the increased assurance of landing with the vehicle nose pointed into the velocity vector, which is critical for the design of the vehicle structure and crew couches to handle the landing impact loads.

Prior to the cancellation of the X-38 program, the guided parafoil system was intensively tested, and had successfully demonstrated the feasibility of the guided parafoil system to land a large vehicle. ${ }^{1,2}$ The parafoil drop test program used subscale and full-scale parafoils. Over 300 drops were conducted using subscale parafoils having a wing area of $750 \mathrm{ft}^{2}$. Using full-scale parafoils, the program conducted twenty drops using $5,500 \mathrm{ft}^{2}$ parafoils and thirteen drops using $7,500 \mathrm{ft}^{2}$ parafoils. The $7,500 \mathrm{ft}^{2}$ parafoil was the final design optimized for the $25,000 \mathrm{lb} C R V$ and demonstrated landings with vertical velocities less than 20 feet per second. The full-scale parafoils were drop tested using standard platforms dropped from cargo aircraft at YPG and using atmospheric test vehicles released from a B-52 aircraft at Edwards Air Force Base. Eight vehicle drops were successfully conducted from the B-52 from altitudes ranging from 23,000 to $40,000 \mathrm{ft}$ mean sea level (MSL). Figure 2 shows the vehicle flying over the target touchdown point at Edwards Air Force Base.

The GN\&C and avionics were demonstrated to have the maturity and robustness required to accurately land the $C R V^{3}$. The testing not only included full-scale parafoil drop tests, but also thirty series of flight tests using the Buckeye powered parachute test vehicle, shown in Fig. 3. The Buckeye was modified by Southwest Research Institute to enable the vehicle to fly autonomously or via remote commands from the ground. The gray box mounted on the deck of the Buckeye, shown in Fig. 3, housed the avionics.

For the flight test operations, the Buckeye was flown to altitude, the engine was turned off, and the X-38 PGNC was activated. The

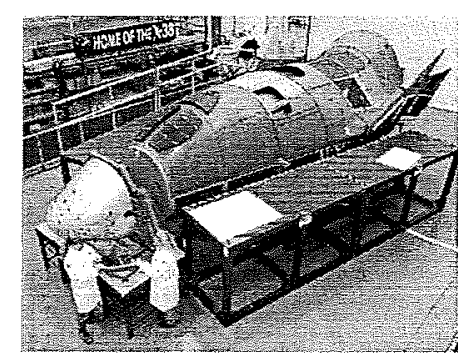

Figure 1. CRV orbital test vehicle under construction

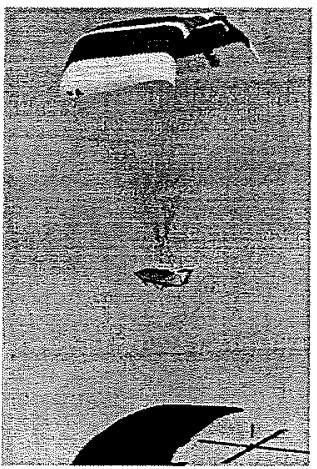

Figure 2. X-38 test vehicle landing near target at Edwards Air Force Base

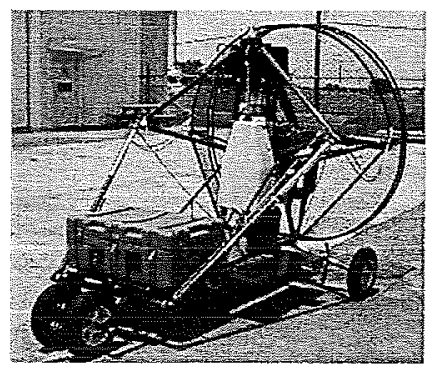

Figure 3. Buckeye modified for autonomous flight 
PGNC then flew the Buckeye autonomously based upon the information loaded into the software for that specific test flight. When a low altitude was reached, the ground pilot restarted the engine via remote commands and flew the Buckeye back up to altitude to begin another PGNC test flight case. Figure 4 shows the Buckeye in flight under a $500 \mathrm{ft}^{2}$ parafoil. Except for the winches, which are canopy specific, all of the PGNC software and hardware used on the platform and atmospheric vehicles was tested using the Buckeye. The Buckeye provided a cost-effective test bed to test hardware and software changes before incorporating them into the more expensive drop test configurations.

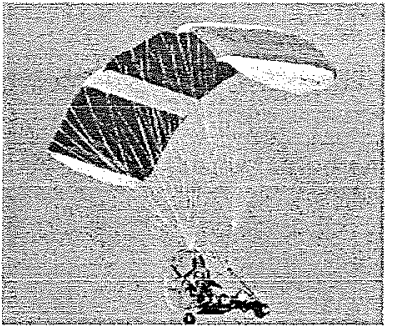

Figure 4. Buckeye in flight

As a result of the successful demonstration of NASA's autonomous, guided parafoil system, Natick Soldier's Center was interested in forming a cooperative effort with NASA to build and test a guided parafoil system for the U.S. Army to deliver $10,000 \mathrm{lbs}$ of useable payload. This cooperative effort is presented in this paper.

\section{U.S Army Guided Parafoil System}

\section{A. Major changes from X-38 design}

NASA's X-38 parachute system was designed to recover a human-crewed spacecraft. The following describes some of the key driving requirements that had to be met.

- Repeatable, low dynamic, on-heading openings

- Space-rated materials

- High design factors

- Increased failure tolerance

- Parachute weight and volume restrictions

- Landing impact velocity and acceleration limits.

Meeting these requirements led to the successful development of a parachute recovery system for the CRV, however, this recovery system is not cost effective for use as a military airdrop system. Because there was growing interest to develop a guided parafoil delivery system in the military, the Natick Soldiers Center and NASA entered into a cooperative effort to reduce the cost of the system and perform drop test demonstrations of that system. Together, NASA and the Natick Soldiers Center identified changes, but due to the short duration of the effort there was not time to significantly alter the design or change the parafoil canopy material. Nevertheless, the changes that were incorporated did provide some reduction in material costs and parafoil assembly time and are summarized below. T. Bennett and R. Fox have presented a more detailed description of these and other changes ${ }^{4}$.

- Reduced the design factors by 20 percent. X-38 required higher safety factors appropriate for humancrewed vehicles.

- Replaced the Kevlar slings and deployment lines with standard Government Furnished Equipment nylon components to reduce cost. The CRV slings were going to be stowed on the spacecraft skin and then covered with insulation for thermal protection during re-entry. Kevlar was selected because it had better tolerance to high temperature and had less bulk to stow under the insulation.

- Replaced the Kevlar parafoil dispersion risers with nylon risers to reduce cost. Kevlar provided weight and volume reductions, which is critical to a spacecraft design.

- Reduced the number of rip-stops in the parafoil. Rip-stops limit the propagation of a tear, thereby minimizing the impact of the tear and the appearance of damage from the ground. Seeing damage from the ground, whether the damage is significant or insignificant, reduces the astronauts' and NASA management's confidence in parachute recovery systems. 
- Returned to the use of continuous instead of fixed length crows feet on the parafoil to minimize time required to make the each crows foot and to attach the suspension lines. A continuous crows foot, shown in Fig. 5, is a single cord with both ends of the cord attached to the canopy. The suspension is attached to the crows foot and can move on the crows foot during dynamic events such as disreefing and control surface deflection. To minimize abrasion between the crows foot and suspension line, fabric buffers are installed.

- Removed the inlets on the lower surface of the parafoil. Ride quality is an important consideration with a crewed spacecraft. As a result, there was a significant amount of effort applied to reduce the deployment dynamics as much as possible, which was one of the biggest challenges of the X-38 program. The program found that controlling the deployment dynamics also contributed to making the deployments more repeatable.

The addition of the first stage inlets was one change that contributed to controlling deployment dynamics. During first stage deployment, parafoil comes out of the deployment bag and has good spreading forces to open the stage. However, without inlets in the lower surface of the first stage, the parafoil tends to lose its shape because the parafoil does not have good forward motion to fill the cells. The parafoil is falling more like a ballistic chute, and the cells are not fully inflated.

When the cell inflation does start, usually one side inflates first, setting up a yawing motion and other adverse dynamics. By adding floor inlets, it takes advantage of the ballistic parachute behavior and quickly, and more importantly, uniformly inflates the stage, minimizing off heading openings and yawing motions. After the cells inflate and the parafoil transitions to forward flight, the ram air entering the leading edge of the parafoil cells closed fabric flaps, sealing off the floor inlets to maintain cell pressurization. Because ride quality is not a consideration for a military airdrop system, the inlets were deleted to simplify manufacturing.

- Replaced symmetrical disreefing with asymmetrical disreefing. This change lowered cost by reducing the number of reefing stages and the time to pack the parafoil.

For symmetrical disreefing, another ride quality associated change in the X-38 program, the same number of cells on each side of the parafoil was opened at the same time. Therefore, when a stage disreefed, cells on both sides were opened at the same time, within the tolerance of the reefing cutters used on each side. By opening the same number of cells on both sides, there was little change to the parafoil heading.

Prior to symmetrical disreefing, asymmetrical disreefing was used, where all of the cells in a stage are on one side of the parafoil. During staging, when a stage on the left side opened, the parafoil yawed left. When a stage on the right side opened, the parafoil yawed right. Therefore during asymmetrical staging, the parafoil yawed left and right as each stage disreefed, inducing dynamics that were translated down to the vehicle. This dynamic motion also increased the chances of getting a twist. A twist could result in failure to separate the confluence fitting. Without confluence separation, the vehicle had reduced roll stability and steering, and the nose would be offset from the vehicle's velocity vector, increasing the change of rollover during landing. Because ride quality is not a consideration for a military airdrop system, asymmetrical disreefing was implemented.

- Mass ratio at brake release was increased. Mass ratio represents the ratio of payload mass to an air mass associated with the canopy, and is defined by the following equation:

$$
\text { MassRatio, } M R=\frac{\text { Mass }_{\text {payload }}}{\rho \times S^{1.5}}
$$


This parameter influences many unsteady parachute phenomena, including inflation and brake release, which are critical phases of parafoil deployment. In X-38, the experience range for a mass ratio for brake release was between 0.56 and 0.84 . Severely adverse dynamics do not occur above mass ratios of 0.4 . Because there does not appear to be adverse dynamics problem at mass ratios above 0.84 , the decision was made to allow mass ratios to be higher than those used in the $X-38$ experience in order to minimize the size, and therefore the cost, of Natick's parafoil. Mass ratios of 1.3 and 1.9 were selected for brake release occurring at 20,000 and 30,000 ft MSL, respectively. Drop testing will provide the opportunity to verify the deployment dynamics at the higher mass ratio.

\section{B. Parachute system description}

The recovery parachute system consists of a $60 \mathrm{ft}$ ringlsot parachute and a $4200 \mathrm{ft}^{2}$ parafoil built by Pioneer Aerospace. The drogue, used during the U.S. Army Guided Precision Air Drop System program and early X-38 testing, is deployed by the extraction parachute and has two stages of reefing (14\% and $42 \%$ of its full open drag area). Disreefing is accomplished using redundant reefing cutters. The time delays for the cutters were selected to manage the inflation loads. After disreefing to full open, the drogue is cut away, using standard strap cutters. The drogue is attached to the vehicle by four slings, and a deployment line is connected to one of the four slings. As the drogue separates from the test article, the deployment line pulls the parafoil deployment bag off of the test article's deck. When the parafoil reaches line stretch, the drogue extracts the parafoil from its bag. The drogue remains attached to the parafoil via an energy modulator that is attached between the deployment sleeve and the upper surface of the parafoil's canopy. ${ }^{5}$ The energy modulator maintains tension on the parafoil suspension system during first stage inflation, resulting in a quick, repeatable spreading of the canopy into first stage. After stroking, the energy modulator separates with one end of the modulator remaining attached to the bag and the other end to the parafoil canopy. During the X-38 program, parafoil deployments were very repeatable with reduced dynamics due to the incorporation of this energy modulator, the lower surface inlets in first stage, and symmetrical disreefing. For the Natick parafoil, only the energy modulator change was incorporated to prevent excessive rebound after line stretch.

The $4200 \mathrm{ft}^{2}$ parafoil, shown in Fig. 6, has a 105.6-foot span, a 40.0 -foot chord, 27 cells, and was derived from the $7500 \mathrm{ft}^{2}$ parafoil developed for NASA's X-38 program. The parafoil has five deployment stages and is fully deployed in 24 seconds. Disreefing is accomplished using redundant reefing cutters and a mid-span reefing technique instead of a slider. During deployment, the parafoil has a single confluence point, which is separated using redundant reefing cutters halfway through deployment for load roll stability. After the parafoil is disreefed to full open, the parafoil. deployment brakes are released using redundant reefing cutters. Initially, the parafoil flies in a circle because it is rigged with a left hand turn. Upon activation, the GN\&C system releases the turn and begins to fly the vehicle autonomously. Should the GN\&C fail to activate, the parafoil would remain in the left hand turn all the way to landing, thereby limiting the footprint of the test article to a predetermined radius that would drift with the prevailing winds.

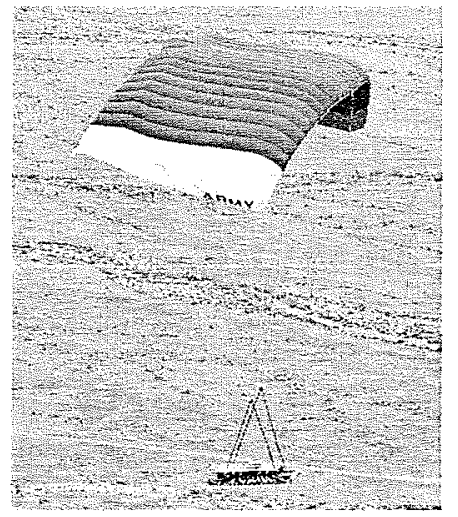

Figure 6. Second drop test using the $4200 \mathrm{ft}^{2}$ parafoil

Because the primary emphasis of the Natick guided parachute program was cost reduction of the parafoil, there was no need to modify the GNC\&C system. Therefore, the NASA GN\&C system, developed during NASA's X-38 program, was used for the drop tests. Southwest Research Institute provided the avionics for the GN\&C system, which consists of winches, laser altimeter, global positioning system (GPS), magnetic compass, barometric altimeter, flight computer, and modems for uplink commands and downlink data. Modified Warn truck winches are used to steer the parafoil and to perform the dynamic flare maneuver. The control surfaces of the parafoil are the outer $25 \%$ of the trailing edge. The control stroke setting is $80 \%$ of the stall stroke setting. The laser, built by Regal of Austria, is the ground proximity sensor used by the GN\&C system to trigger the flare. A Trimble GPS card provides the primary navigation parameters (i.e. position, altitude and heading data) for the GN\&C software. In the event of a GPS failure, the software navigates by dead reckoning using the compass and barometric altimeter data. The flight computer interfaces with the sensors and effectors and hosts the PGNC.

The PGNC was built by Astrium Aerospace, which is associated with the European Space Agency. NASA modified and improved the software during the $\mathrm{X}-38$ flight test program. The basic approach of the PGNC is not 
unlike most GN\&C designs. ${ }^{3}$ The PGNC logic develops a reference trajectory to reach the target, uses guidance to make trajectory adjustments in response to performance and flight conditions, uses navigation to determine its location, and uses control logic to fly the reference trajectory. The reference trajectory is shown in Fig. 7.

The first guidance phase, shown in Fig. 7, is the target acquisition turn. The guidance then exits the turn and proceeds to a homing leg, a straight flight portion headed towards the energy management circle (EMC) entry point. The next phase is the EMC entry turn. While on the EMC, the guidance modulates the diameter of the EMC diameter to regulate range error caused by wind or parafoil performance errors. At the predetermined heading for final, guidance initiates an EMC exit turn. The final heading is typically established by the winds of the day, so the parafoil can land into a headwind. On final approach, the parafoil continues to minimize cross track error. Ten seconds prior to flare, a pre-flare setting of $40 \%$ flaps is commanded to minimize dynamics before going into flare. When the laser altimeter detects the predetermined altitude, the PGNC initiates the flare, and the parafoil flaps are reeled in to $100 \%$ at about 1.5 feet of control line per second.

The PGNC can autonomously fly the entire flight profile from built in turn release to landing, but the PGNC is also equipped with a receiver that allows a ground station to transmit real time user input parafoil control surface commands, referred to as manual mode. Manual mode has been used to provide manual override of the PGNC, if required, and to perform an extensive series of parafoil maneuvers in order to determine the aerodynamic characteristics of the parafoil. ${ }^{67}$ These maneuvers include flap settings, flares at altitude, turns using various combinations of differential control surface settings, and stall maneuvers. The PGNC also has a transmitter to transmit PGNC data to the ground station to perform real-time monitoring of the GN\&C system and the test article's ground track.

Mission planning to select the "green light" release coordinates is done using the PGNC mission planner and a footprint tool. These tools use winds aloft data obtained from balloons released at the drop site to select a release point that not only ensures that the load can reach the designated target point but also ensures that the released parachutes remain on the range. The release and target points, landing heading, the winds aloft data and other PGNC data are loaded into the flight computer prior to the drop aircraft's takeoff.

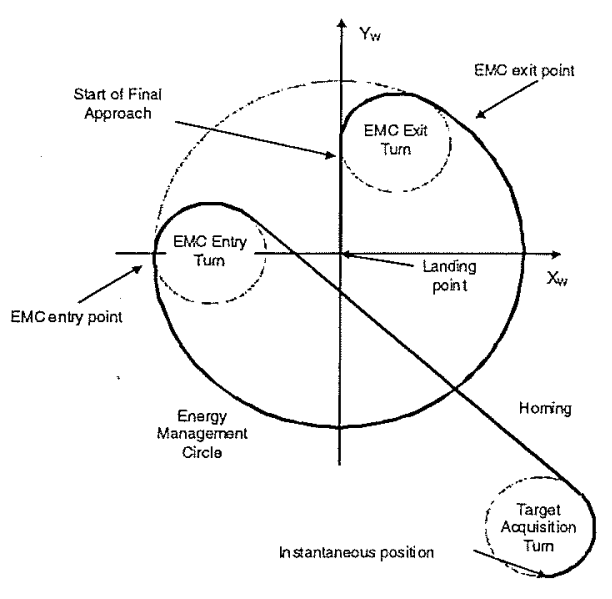

Figure 7. PGNC reference trajectory

\section{Drop Test Description}

The purpose of the drop test program was to demonstrate an autonomous flight using a guided parafoil system to deliver 10,000 lbs of useable payload and land softly using a dynamic flare. The test article's rigged weight was $15,700 \mathrm{lb}$ at extraction. The drop test payload consisted of a standard 20-foot Type $\mathrm{V}$ airdrop platform, a standard 12-foot weight tub, recovery parachutes, an instrumentation system, and a PGNC system. Standard YPG range services used included Kineto Tracking Mounts for tracking data, ground-to-air and on board video, still photography, and parachute and load post-drop recovery.

Instrumentation installed on the load was used to gather data to validate simulation models, preflight loads predictions, and post flight trajectory reconstruction. The YPG-installed instrumentation included strain links for the drogue and parafoil sling load and a differential GPS for test article tracking information. The NASA instrumentation, installed and checked by Lockheed Martin-Houston, included an air data probe (ADP), an accelerometer package, an IST impact recorder, winch battery voltages and currents, a data collection and recording system, an upward-looking video camera and recorder, and load cells on the deployment lines and on the parafoil risers, crossover slings, control lines, and leading edge of the canopy's lower surface ${ }^{1}$. The instrumentation was developed over the forty-two X-38 full-scale parafoil LVAD drop tests.

Another type of instrumentation developed by NASA is the parafoil inclinometer system ${ }^{8}$. This system uses accelerometers attached to the lower surface of the parafoil canopy. Data from the accelerometers is collected and stored in a data logger, also located on the canopy, and downloaded after the drop. The data, valid during steady state flight, measures the parafoil's trim angle of attack. Trim angle of attack is important to predict, evaluate and optimize a parafoil's flight performance. 
A helicopter was used to fly chase to assist in identifying problems with the parafoil not visible from the ground, provide real-time assessment of the parafoil and PGNC performance, and to obtain video and photography for documentation and post drop analysis.

In the X-38 program, simulation models were developed by NASA to support design development, provide pre-drop performance predictions, and perform post-drop performance analysis and trajectory reconstruction. These models were validated during the X-38 drop test program and used on the Natick parafoil program. One of the models is the decelerator system simulation (DSS) ${ }^{9}$.

The DSS is a six-degree of freedom (6-DOF) model of a round parachute and a 6-DOF model of the vehicle. This model is used to predict the drogue sling loads during inflation; parachute disreef event timing, dynamics and inflation loads; and over-rotation during payload extraction from cargo aircraft. The over-rotation simulation was developed to bound the acceptable test article center of gravity (CG) for the platform configuration. If the $\mathrm{CG}$ is too far aft, the platform will over-rotate immediately upon extraction from the aircraft and can result in the load being upside down, causing the riser to strike the load or become entangled with the load. Damage to the riser could lead to riser failure and loss of the load. Due to this concern, the X-38 developed the weight and balance for each load to ensure that the over-rotation was 120 degrees or less. The assembly of the load was modeled using a spreadsheet identifying the location of the components and tub weights. The weight and CG of the load was verified prior to being loaded onto the C-130.

Another significant model is the parafoil dynamics simulator (PDS), which is a 6-DOF rigid body parafoil and a 2-DOF rigid body vehicle model, coupled at the confluence point of the system. ${ }^{8}$ The PDS models a parafoil and vehicle system during all phases of flight. These phases include parafoil inflation and transition to forward flight; stage disreefing and brake release; PGNC flight; manual flight maneuvers; and flare and touchdown. This model is used in the preflight timeframe to derive predictions on expected parafoil performance; parafoil flight time and ground track; and flare performance; and to develop the test plan for manual maneuvers. Parafoil aerodynamic characteristics, such as glide ratio, turn performance, and flare timing, are derived from the model and then incorporated into the PGNC to optimize GN\&C performance. During many of the X-38 drop tests, planned manual maneuvers were flown to gather aerodynamic data to validate the PDS. After a drop test, the PDS is used determine the longitudinal and lateral-directional aerodynamics and for flight reconstruction to evaluate PGNC and flare performance.

Finally, another model developed during the $\mathrm{X}-38$ program was the Brinkley model to assess the effect of the landing impact on a deconditioned crewperson. The model is based upon one developed by Wright Patterson Air Force Base to analysis ejection seat human tolerances. The input is filtered high impact accelerometer recorded during landing, and the output is criteria for the risk of injury as a function of dynamic response of the loads. This model was used to compare the test results with the X-38 test program results.

\section{Drop Test Results}

Three drop tests were conducted. The test article, weighing 15,700 lbs, required a $22 \mathrm{ft}$ extraction parachute and was dropped from a cargo aircraft at 17,900 ft MSL. For each test, the load was built up within the CG limits to minimize over-rotation following load extraction from the aircraft. The over-rotation was less than the predicted value of 120 degrees for all three drops.

The primary test objectives were to demonstrate a derivative of the X-38 parafoil recovery system for use as a precision aerial delivery system to deliver $10,000 \mathrm{lbs}$ of useable payload; to demonstrate acceptable drogue and parafoil deployment, disreef and performance; to verify parafoil full open aerodynamic performance; and to demonstrate the parafoil recovery process without using a crane. By the third drop test, all test objectives had been successfully demonstrated. The results of each test are summarized below.

\section{A. Drop test 1}

Because this was the first drop of a new parafoil, a test plan was developed to gather aerodynamic data during parafoil flight for validation of the PDS aerodynamic database by manually executing planned longitudinal and lateral-directional maneuvers. After completion of the maneuvers, the test team had planned to command the PGNC back to autonomous mode for landing.

However, a significant anomaly occurred during the drop, which prevented the test maneuvers from being performed. The crows feet in the $2^{\text {nd }}$ and $3^{\text {rd }}$ stages failed following the disreef event for each stage. The damage was difficult to see from the ground, but the chase helicopter was able to confirm the failure. The test team used the autonomous mode for the PGNC to fly the test article into the wind for landing and then commanded manual mode to prevent the software from executing the flare. To reduce the landing speed, the team commanded the 
flaps to $50 \%$, resulting in a vertical velocity at touchdown of 20 feet per second. In spite of the failure, the parafoil safely landed the test article below the target landing velocity.

Post drop inspection of the failed crows feet indicated that the buffers were not effective in preventing abrasion between the suspension line and crows feet. In fact, the abrasion was actually occurring between the crows foot and the inside surface of the buffer when the suspension line shifted on the crows foot during deployment and trailing edge deflection. Re-evaluation of the parafoil design changes between X-38 and the Natick programs revealed that the configuration and spacing of the crows feet were different, resulting in an increase of movement of the suspension line on the crows feet. In the Natick parafoil, there were a smaller number of crows feet and an increased spacing between the crows feet V-tabs, creating a different load distribution that the crows feet had to handle. For the second drop, the continuous crows foot design was replaced with the design developed and tested successfully on the X-38 parafoils, which was to use individual crows foot. Individual crows feet consist of two separate cords each of which are attached to the canopy and then joined together with the suspension line. At this juncture, a buffer is installed. The strength of the cord used to construct the crows feet was also increased.

During parafoil deployment, there was a pronounced parafoil surge during the transition from ballistic to forward flight in first stage, indicating the deployment brakes may not be optimized for the new parafoil. No change was incorporated for the second drop because the surge was not severe enough to adversely affect the dynamics. The setting would be re-evaluated on the second drop test.

No anomalies were identified with the GN\&C system. All of the software, sensors and effectors functioned properly. No software changes were needed for the second drop.

PDS predicted the parafoil lift over drag (L/D) to be 3.55 at $0 \%$ control setting, which is an improvement over the X-38 parafoil which was 3.0. This improvement was due to the smaller number of suspension lines, resulting in reduced line drag. During the drop test, a bump was detected near the trailing edge and extended across the entire span of the canopy. This bump is due to a chordwise compression near a line group and is referred to as reflex. As a result of this reflex, the prediction for $L / D$ was adjusted down to 3.26. Verification of the $L / D$ was not possible on this drop due to the crows foot failures.

\section{B. Drop test 2}

As in the first drop test, a test plan was developed was to conduct manual maneuvers to gather aerodynamic data on the parafoil performance. In addition, active control of the parafoil was going to be returned to automatic mode after completing the test maneuvers so that the PGNC could develop and execute a trajectory to reach the target and perform a flare.

The modification to the crows foot configuration solved the abrasion problem that had occurred on the first drop. As a result, parafoil performance was excellent allowing all planned manual maneuvers to be completed. The maneuvers that were executed are described below. The percentage of deflection is given for the left and right control surfaces. Each maneuver is held for a pre-determined number of seconds to ensure that dynamics induced as the result of the maneuver have damped out.

- $50 / 50 \%$ flaps for 25 seconds

- $80 / 80 \%$ flaps for 25 seconds

- $100 / 100 \%$ flaps for 25 seconds

- $0 / 0 \%$ for 30 seconds

- $\quad 0 / 70 \%$ turn for 20 seconds

- $0 / 80 \%$ turn for 15 seconds

- $0 / 90 \%$ turn for 15 seconds

- $0 / 100 \%$ turn for 15 seconds

- $0 / 0 \%$ for 25 seconds

- $0 / 15 \%$ turn for 25 seconds

After the maneuvers were completed, the PGNC was commanded back to automatic mode. The PGNC developed a trajectory, flew the load towards the landing target and initiated the flare, landing approximately 1,400 feet short of the target. The flare achieved a vertical landing velocity of 10 feet per second and a horizontal landing speed of 46 feet per second. The ground track is shown in Fig. 8. 


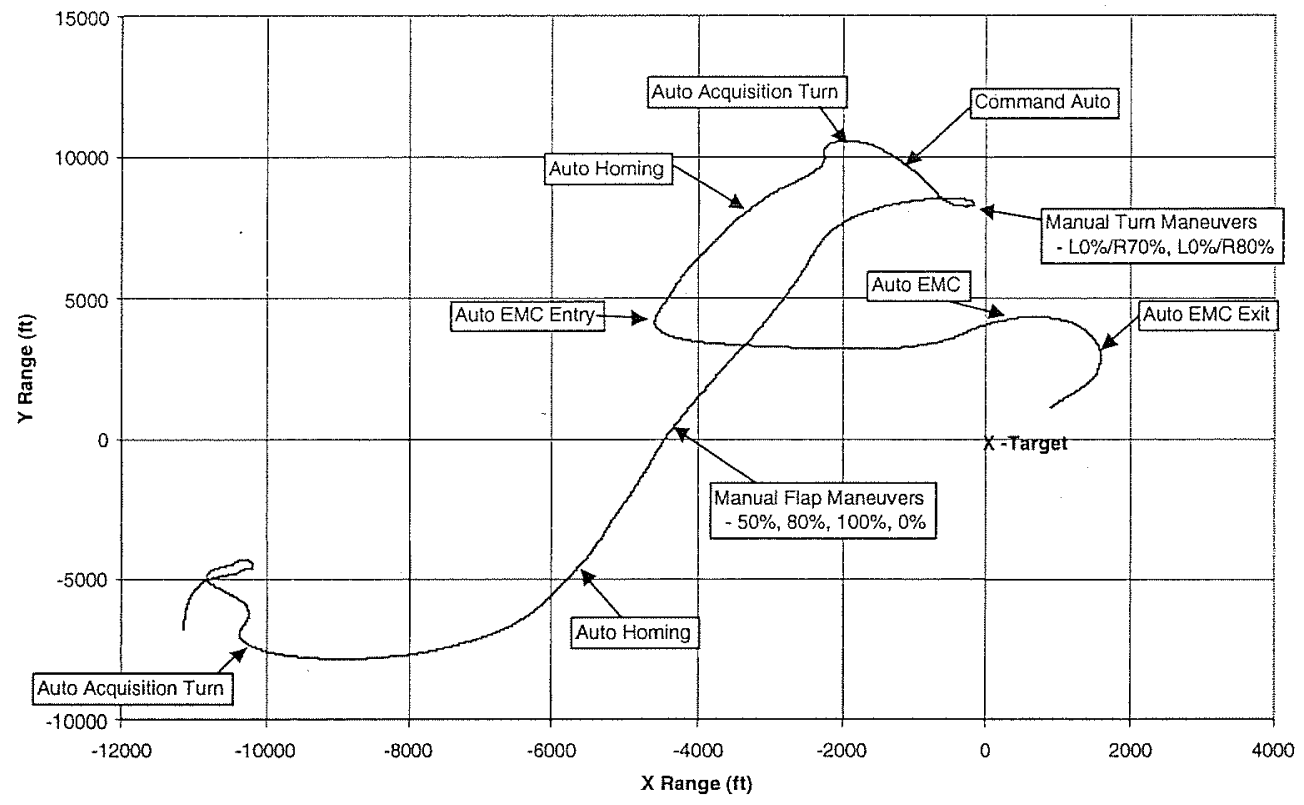

Figure 8. Ground track of second drop test

Post drop analysis of the PGNC revealed that the landing was short because PGNC was not moded from manual to automatic soon enough and because the guidance did not compensate correctly for the extended final. By turning control over to the PGNC too late, the PGNC did not have sufficient altitude to perform the needed energy management to reach the target. Guidance modulates the pattern size for this energy management, including the expected length of the final for the approach to the target, however, guidance was not programmed to compensate for the wind. A software change for the third drop was to allow the final extension distance to be modulated with the energy management logic.

Another change, which would improve landing accuracy on the third drop, was to increase the maximum parafoil control deflection from $80 \%$ to $90 \%$ of stall stroke, allowing the PGNC to make more aggressive turns and thus improving turn performance for energy management. The manual maneuver at $100 / 100 \%$, performed on the second drop, confirmed that parafoil would not stall when commanded to the maximum control deflection, thus ensuring the software could not stall the parafoil.

Figures 9 and 10 are plots of the coefficient of lift $\left(\mathrm{C}_{\mathrm{L}}\right)$ and coefficient of drag $\left(C_{D}\right)$, which show that lift and the drag increase as the control line deflection increases. As the control line deflection is decreased below $15 \%$, there is little effect on lift or drag, because the control lines are being pulled in, but the trailing edge is no longer being deflected. This is due to reflex, which is caused by a deformation of the trailing edge when it is not loaded by the control lines.

These plots compare flight data obtained during the second drop with the PDS model data, including models for the $4200 \mathrm{ft}^{2}$ parafoil as well as for the 7500 $\mathrm{ft}^{2}$ parafoil. For the $7500 \mathrm{ft}^{2}$ parafoil, two different rigging angles (RA), 8 degrees and 10 degrees, and two different suspension line materials, Kevlar and Zylon, were tested. The weight to strength ratio for Zylon is less than Kevlar, resulting in less parafoil suspension line drag and weight. The final configuration for the X-38 parafoil was the $7500 \mathrm{ft}^{2}$ parafoil with a RA of 8 degrees and Kevlar suspension lines.

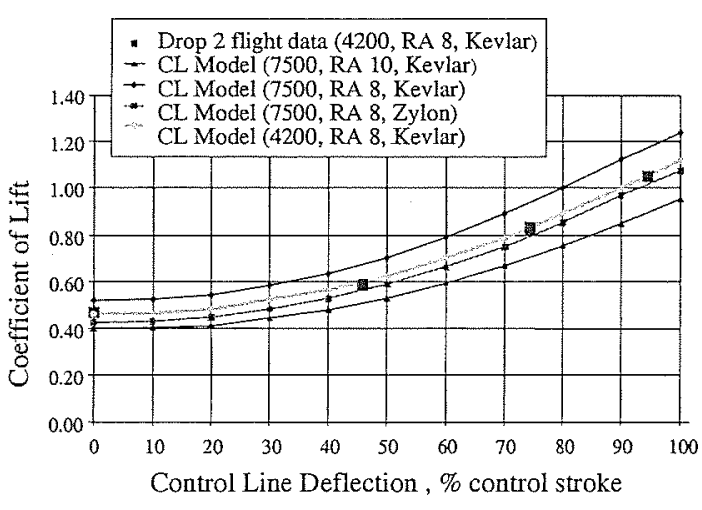

Figure 9. Coefficient of lift for $4200 \mathrm{ft}^{2}$ parafoil 
The data points obtained during the second drop of the $4200 \mathrm{ft}^{2}$ parafoil match well with the PDS model for that parafoil, demonstrating the maturity of the model and the scalability of the parafoil aerodynamics.

Figure 11 is a plot of the lift to drag ratio (L/D) versus control line deflection and shows that $L / D$ decreases with increasing control line deflection. The $\mathrm{L} / \mathrm{D}$ was higher than the final configuration of the $\mathrm{X}$ $387500 \mathrm{ft}^{2}$ parafoil, which has a RA of 8 degrees and Kevlar suspension lines. As shown in the figure, the L/D for the $4200 \mathrm{ft}^{2}$ parafoil at $0 \%$ control line deflection is 3.37 , which is higher than the preflight prediction of 3.26 . The reason for the higher $L / D$ is that the actual parafoil drag and the trim angle of attack were lower, resulting in increased $L / D$ and velocities. The trim angle of attack was predicted preflight to be 6.0 , and was measured by the parafoil inclinometer to be 5.75 degrees. The vertical and horizontal velocities are shown in figures 12 and 13.

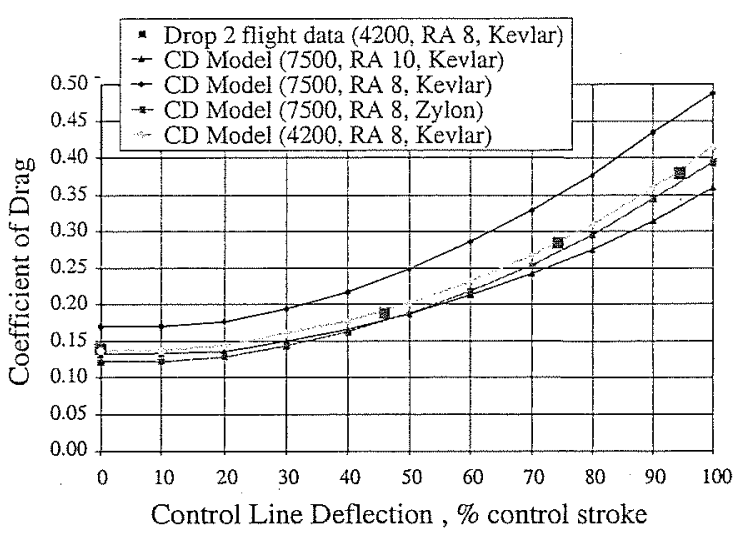

Figure 10. Coefficient of drag for $4200 \mathrm{ft}^{2}$ parafoil

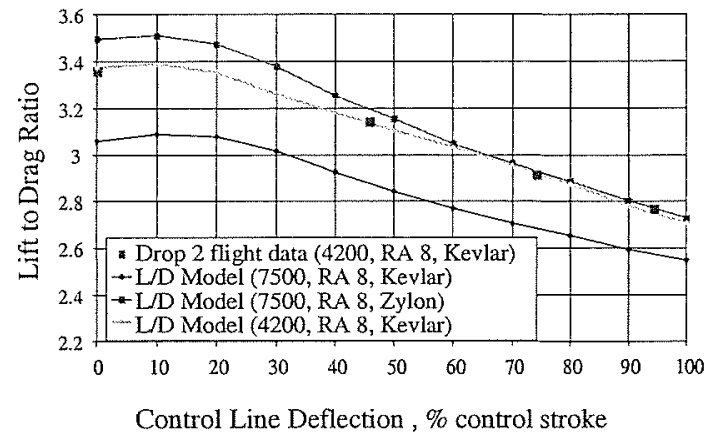

Figure 11. Lift to drag ratio for $4200 \mathrm{ft}^{2}$ parafoil

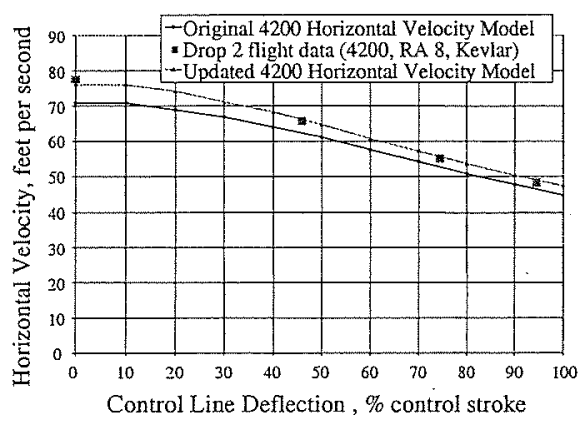

Figure 13. Horizontal velocity
versus control deflection

\section{Drop test 3}

Third drop was conducted as part of the PATCAD. For this demonstration, the drop was autonomous. The overall recovery system performance was excellent and met all test objectives. As experienced in the first drop, there was pronounced coning motion during the parafoil's transition from ballistic to forward flight, resulting in some twist, which did clear during the deployment sequence. While the dynamics may be more than desired for ride quality considerations of a human-crewed spacecraft, the dynamics pose no problem for a cargo delivery application. The dynamics can be minimized on further drop tests by fine-tuning the deployment brake setting. 
Following deployment, the parafoil was in a left hand turn. After an onboard timer elapsed, the GN\&C was activated and the entire flight was autonomous. The GN\&C calculated a reference trajectory and commanded the winches to fly that trajectory. Using the laser altimeter, the GN\&C commanded the flare maneuver. The vertical and horizontal landing speeds were 10 feet per second and 59 feet per second, respectively. The payload came to rest 206 feet from the intended target, the smallest miss distance in the X-38 test program. As part of the demonstration, the parafoil was recovered without the assistance of a crane. The recovery crew of about seven persons was able to recover the parafoil and load it into a pickup truck in approximately one hour.

\section{Conclusions}

The X-38 program spent several years developing a guided parafoil system to recover a human-crewed spacecraft. The major challenges associated with developing such a system included deploying large parafoils, making the deployments repeatable, reducing dynamics associated with deployment, flying accurately to the landing target and landing softly. The X-38 test program successfully demonstrated that these challenges can be overcome. NASA's cooperation with Natick also demonstrated that the guided parafoil system is feasible to develop as a military aerial delivery system and that the cost of the system can be reduced. Further modifications are possible to make the guided parafoil system meet the cost targets established by Natick.

\section{Acknowledgments}

The authors thanks the following individuals for their contributions to this test program: Brian Anderson, the NASA X-38 Program Manager; Richard Benney and Ed Doucette of Natick for supporting this cooperative drop test program; Ricardo Machin, the NASA X-38 Parafoil Test Director; Tom Bennett, Roy Fox, et al of Pioneer Aerospace for modifying the design in a short time to meet the Army's requirements and delivering top quality products; Dean Wolf for giving an independent, objective view and keeping us honest; Don Reed for his excellent work in the chase helo; Jeff Fox for the great job of activating all of the instrumentation before the payload is extracted from the aircraft; Wayne Petterson for his calm, cool manner as parafoil pilot; Pete Cuthbert for his valuable computer modeling using the DSS; Ron Sostaric for his excellent operation of the Buckeye and support in delivering the best GN\&C system to fly a parafoil and the best ground station; Dave Ogden, Steve Spence, and Carl Bargainer of Southwest Research Institute for delivering top quality GN\&C changes on time regardless of the short schedule given to accomplish the work; Bernie Rosenbaum for solving so many mechanical problems and making sure the winches and batteries will work and fly the parafoil; Paul Royall and Bobby Jennings of Lockheed Martin-Houston for providing instrumentation expertise; Dale Jennings of Lockheed-Martin Houston for providing his rigging expertise to the team and for getting up early on drop mornings to set up the forward ground station; and Ray Schultz and Becky Brocato of Yuma Proving Ground for their tireless assistance in making this rapid test series possible.

\section{References}

${ }^{1}$ Machin, R., Stein, J., and Muratore, J., "An Overview of the X-38 Prototype Crew Return Vehicle Development and Test Program”, AIAA-99-1703, 15 "he CEAS/AIAA Aerodynamic Decelerator Systems Conference, pages 20-37, June 1999.

${ }^{2}$ Bennett, T. and Fox, R., "Design, Development \& Flight Testing of the NASA X-38 7,500 Ft Parafoil Recovery System", AIAA-2003-2107, 16 ${ }^{\text {th }}$ CEAS/AIAA Aerodynamic Decelerator Systems Conference, May 19-22, 2003

${ }^{3}$ Strahan, A., "Testing of Parafoil Autonomous Guidance, Navigation \& Control for X-38", AIAA-2003-2115, 16 ${ }^{\text {th }}$ CEAS/AIAA Aerodynamic Decelerator Systems Conference, May 19-22, 2003

${ }^{4}$ Bennett, T, and Fox, R., "Design, Development \& Flight Testing of the U.S. Army $4200 \mathrm{Ft}^{2}$ Parafoil Recovery System", AIAA-2005-1628, $18^{\text {th }}$ CEAS/AIAA Aerodynamic Decelerator Systems Conference, May 2005

${ }^{5}$ Bennett, T. and Smith, J., and Fox, R., "Testing and Development of the NASA X-38 Parafoil Upper Surface Energy Modulator", AIAA-99-1753, 15 ${ }^{\text {th }}$ CEAS/AIAA Aerodynamic Decelerator Systems Conference, June 1999

${ }^{6}$ Inacomini, C. and Cerimele, C., "Lateral Directional Aerodynamics from a Large Scale Parafoil Test Program", AIAA-99$1731,15^{\text {th }}$ CEAS/AIAA Aerodynamic Decelerator Systems Conference, June 1999

${ }^{7}$ Inacomini, C. and Cerimele, C., "Longitudinal Aerodynamics from a Large Scale Parafoil Test Program", AIAA-99-1732, $15^{\text {th }}$ CEAS/AIAA Aerodynamic Decelerator Systems Conference, June 1999

${ }^{8}$ Madsen, C. and Cerimele, C., "Flight Performance, Aerodynamics, and Simulation Development for the X-38 Parafoil Test Program", AIAA-2003-2108, $16^{\text {th }}$ CEAS/AIAA Aerodynamic Decelerator Systems Conference, May 19-22, 2003

${ }^{9}$ Cuthbert, P., "A Software Simulation of Cargo Drop Tests", AIAA-2003-2132, $17^{\text {th }}$ CEAS/AIAA Aerodynamic Decelerator Systems Conference, May 2005 